\title{
Mapping piezoelectric response in nanomaterials using a dedicated non-destructive scanning probe technique
}

\author{
Yonatan Calahorra ${ }^{1}$, Michael Smith ${ }^{1}$, Anuja Datta ${ }^{1}$, Hadas Benisty ${ }^{2}$, Sohini Kar-Narayan ${ }^{1 *}$ \\ ${ }^{1}$ Department of Materials Science and Metallurgy, University of Cambridge, 27 Charles Babbage \\ Road, Cambridge CB3 OFS, UK \\ ${ }^{2}$ Department of Electrical Engineering, Technion-ITT, Haifa 3200003, Israel \\ email:sk568@cam.ac.uk
}

There has been tremendous interest in piezoelectricity at the nanoscale, for example in nanowires and nanofibers where piezoelectric properties may be enhanced or controllably tuned, thus necessitating robust characterization techniques of piezoelectric response in nanomaterials. Piezo-response force microscopy (PFM) is a well-established scanning probe technique routinely used to image piezoelectric/ferroelectric domains in thin films, however, its applicability to nanoscale objects is limited due to the requirement for physical contact with an atomic force microscope (AFM) tip that may cause dislocation or damage, particularly to soft materials, during scanning. Here we report a non-destructive PFM (ND-PFM) technique wherein the tip is oscillated into "discontinuous" contact during scanning, while applying an AC bias between tip and sample and extracting the piezoelectric response for each contact point by monitoring the resulting localized deformation at the AC frequency. ND-PFM is successfully applied to soft polymeric (poly-L-lactic acid) nanowires, as well as hard ceramic (barium zirconate titanatebarium calcium titanate) nanowires, both previously inaccessible by conventional PFM. Our NDPFM technique is versatile and compatible with commercial AFMs, and can be used to correlate piezoelectric properties of nanomaterials with their microstructural features thus overcoming key characterisation challenges in the field.

Piezoelectricity, by which certain materials can directly inter-convert mechanical and electrical energy, may be considerably enhanced by reducing dimensionality and/or size ${ }^{1-6}$, with many applications in energy harvesting ${ }^{7-11}$ and sensing ${ }^{12-14}$. In light of the many potential applications of piezoelectric nanomaterials, there is a crucial need for robust characterization techniques that can establish the relationship between structure and piezoelectric properties at the nanoscale. In this regard, piezo-response force microscopy (PFM) ${ }^{15}$, in which an AFM tip is scanned across a sample in contactmode and the mechanical deformation of the surface in response to an applied tip-sample bias voltage is recorded, has been the standard workhorse for the imaging and characterization of piezoelectric/ferroelectric domains in thin films ${ }^{15-23}$. However, its role in characterizing piezoelectricity 
in nanoscale objects, such as nanowires and nanoparticles, has been far more limited, being mostly restricted to single-point measurements of piezoelectric deformation as a function of tip-sample bias. This is because the continuous contact required between tip and sample during PFM imaging often causes damage to such nanoscale objects, or even dislocation. Hence, "mapping" of piezoelectric properties in nanomaterials, such as spatially resolved piezoelectric coefficients $\left(d_{\mathrm{ij}}\right)$, is seldom reported. In order to circumvent this problem, we have developed a "non-destructive" PFM (ND-PFM) technique that allows spatially resolved piezoelectric properties of nanomaterials to be directly measured. We demonstrate the versatility of our method in addressing a wide range of piezoelectric nanomaterials by applying it to both polymeric as well as ceramic nanowires ${ }^{24,25}$ which are otherwise destroyed or dislodged when using conventional PFM.

In the ND-PFM mode, the tip is oscillated into intermittent contact with the sample during scanning, while an AC voltage is simultaneously applied between the tip and the sample, and the piezoelectric response at the $\mathrm{AC}$ frequency, i.e. the deformation of the sample in response to the applied bias across it, is extracted for each contact event using high-speed data capture and analysis techniques. As in contact-mode PFM (c-PFM), the piezoelectric response may arise in three orientations: out-ofplane, and two orthogonal in-plane deformations, corresponding to different piezoelectric coefficients, $d_{\mathrm{ij} .}$. The piezoelectric response is therefore measured in terms of both the vertical and lateral signal from the photodetector. Note that is not straightforward to extract the piezoelectric coefficients from these signals without prior knowledge of the polarization orientation relative to the cantilever, as will be discussed in the next section. The two fundamental principles manifested in ND-PFM are (i) the ability to perform PFM measurements in a non-contact mode, minimizing damage to delicate samples, and (ii) acquiring data which is physically relevant, i.e., obtained at times when tip and sample are in contact. These principles have guided a previous study by Rodriguez et al., where a similar concept was explored in "intermittent-contact" PFM (IC-PFM) ${ }^{26}$ which was only applicable in liquid environments. Rodriguez et al. used tapping mode AFM (in the fundamental frequency of the cantilever) for obtaining topographic data while reducing tip-sample interactions, overlaid by an electrical AC drive at the contact resonance frequency (close to the second fundamental mode of the cantilever), in order to obtain physically relevant data predominantly when tip and sample are in contact. In stark contrast to the NDPFM method presented here, it was shown that IC-PFM is effective only while applied in a liquid environment which was specifically required to achieve the attenuation of the locked-in signal when the tip and sample are not in contact. This mode of operation brings about many practical difficulties, such as the requirement of a liquid cell for imaging, as well as fundamental implications, e.g. in instances where the probed material and the liquid environment interact ${ }^{26}$. Indeed, to the best of our knowledge, the IC-PFM method has not been subsequently used to image nanomaterials, and is fairly restrictive in its implementation. On the other hand, our ND-PFM technique is shown to work under 
ambient conditions, and is compatible with commercial AFMs, making it a versatile, powerful and accessible technique that can be used to image piezoelectric properties of a wide range of nanomaterials.

\section{Results and discussion}

\section{Demonstration of ND-PFM using standard calibration sample}

We demonstrate the concept of ND-PFM and our implementation of it starting with a standard periodically poled lithium niobate (PPLN) PFM calibration sample (PFM-SMPL, Bruker, $d_{33}=7.5 \mathrm{pm}$ $\mathrm{V}^{-1}$ ). Figure 1 schematically shows conventional contact-mode PFM (c-PFM) and ND-PFM measurement set-ups as implemented in a standard AFM apparatus. In c-PFM (Figure 1a), an ac bias, $V_{\mathrm{ac}}$, is applied between the AFM tip and a conductive substrate, such that an ac electric field is generated across the PPLN sample, as contact is maintained between the tip and sample while scanning via a constant force feedback mechanism that controls the $z$-piezo motor (point 1). The photodiode signal is directly fed to a lock-in amplifier, whose output is the PFM signal (point 2), in either in-phase/out-ofphase, or amplitude/phase component configuration. Both vertical and lateral deflections of the AFM cantilever can be monitored corresponding to the out-of-plane and in-plane piezoelectric responses of the sample, respectively. In this case, the vertical signal corresponds to the $d_{33}$ coefficient, while the lateral signal cannot be directly related to a single coefficient. Alternatively, in the so-called "peakforce" mode (see schematic in Supporting Information Figure S1), the vertical position of the tip is oscillated via the $z$-piezo motor $(0.25-2 \mathrm{kHz})$ such that it makes discontinuous contact while performing periodic indentations on the sample (point 3$)^{27}$. The tip is simultaneously raster-scanned at a lower frequency $(\sim 1 \mathrm{~Hz})$ across the sample, thus minimizing the tip-sample contact time while monitoring force vs. distance curves on the fly. The peak-force mode provides the basis for our ND-PFM technique, as shown in Figure 1b, where $V_{\text {ac }}$ is superimposed on the tip at a much higher excitation frequency than the mechanical oscillations. The PFM signal is extracted for each contact point on the scan directly from the tip deflection at the excitation frequency (point 4), only during the time period in which the tip and sample are in contact as confirmed from the force vs. distance curves. This is done by applying a digitalized (virtual) lock-in (L.I) procedure to the data, at the excitation frequency. In this way, the piezoelectric response of the sample can be spatially resolved and quantified while taking advantage of the non-destructive benefits nature of intermittent contact operation. 
(a)

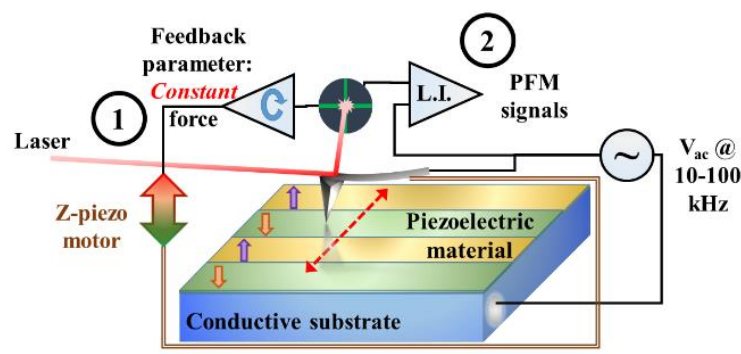

(b)

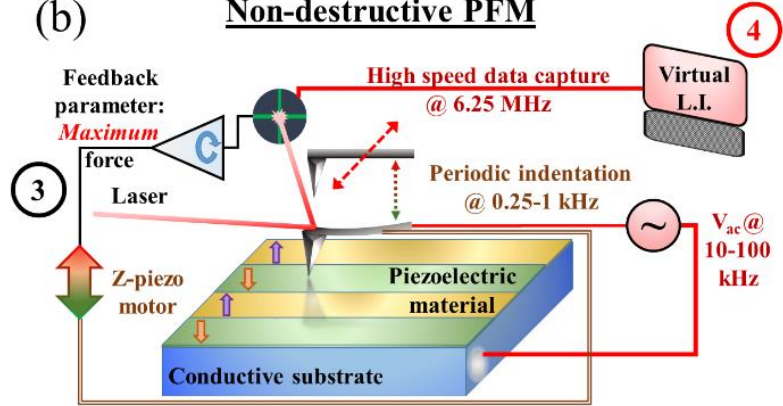

Figure 1. Schematic of PFM operation and signals: a) Standard contact mode PFM; b) Intermittentcontact PFM. Red colour/tint indicates modifications made atop the peakforce mode, added in order to induce and extract PFM signals, via a digitalized virtual lock-in (L.I) procedure. Interest points 1-4 draw the attention to different parts of the schematic, referred to in the main text, and in Figure 2. Doubled brown lines indicate mechanical control, while black/red single lines indicate electrical signals.

$V_{\text {ac }}$ was applied between the tip and sample (in our case the tip is grounded) at a frequency, $f_{\mathrm{PFM}}$ $\sim 100 \mathrm{kHz}$, resulting in piezoelectric deformations when the tip contacted the sample. During scanning, the tip deflection (both vertical and lateral on separate channels) was recorded using high-speed data capture (@6.25 MHz) for a duration of about $2 \mathrm{~s}$, made to correspond to a single horizontal image line by tuning the scanning size and speed. This signal was then digitally processed to extract the frequency component corresponding to $f_{\mathrm{PFM}}$, as detailed in Supporting Information Figure S2. Importantly, this 'virtual lock-in' procedure was performed only on the data obtained when the tip and sample were in contact (see Figure 2b), thus avoiding averaging of the signal throughout the mechanical oscillation period, which would be the outcome of simply displaying the standard PFM lock-in channel output (see Supporting Information Figure S3 and related discussion). As in the case of c-PFM, we extracted both the in-phase (X channel) and out-of-phase (quadrature or $\mathrm{Y}$ channel) piezoelectric response value corresponding to the spatial location of each force vs. distance curve. A value is obtained for vertical and lateral signals separately. 


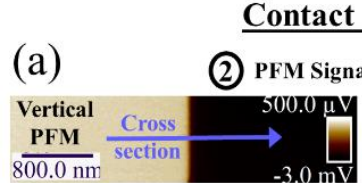

(c)

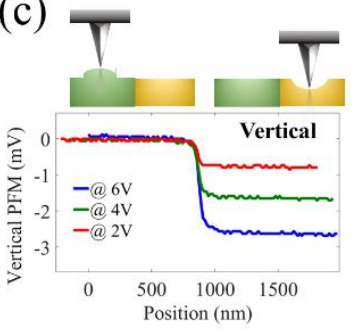

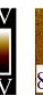

\section{$\frac{\text { PFM }}{800.0 \mathrm{n}}$}
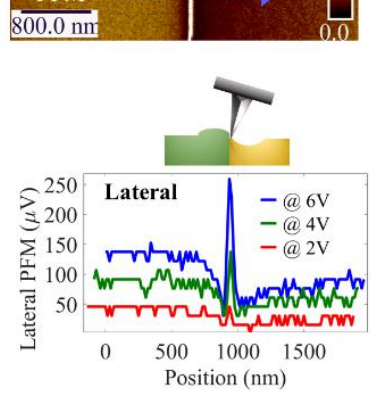

Non-destructive PFM

(b)

(d)

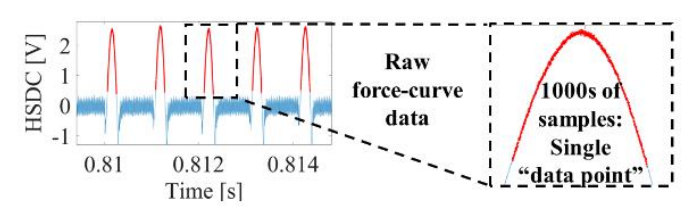

(4) Virtual lock-in output (a) $f_{P F M}$

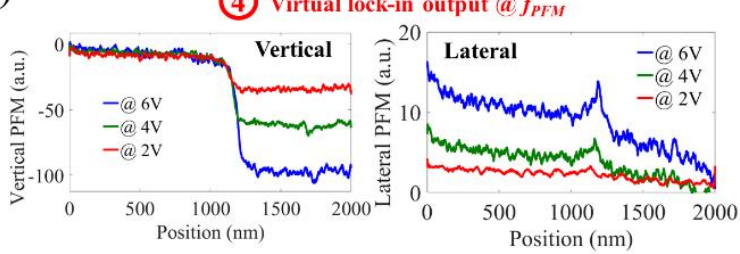

Figure 2. Representative signals obtained from a PPLN sample for c-PFM (left) and ND-PFM (right) for $V_{\mathrm{ac}}=2,4,6 \mathrm{~V}$ : a) vertical and lateral c-PFM images across a boundary; b) a section of HSDC force curves obtained during ND-PFM, while scanning a line on the same area as in [a]. The contact-time data is marked red (inset shows zoom-in to a single curve); c) cross-sections taken from the images in [a]. Upper insets schematically show the tip deflection when on the two oppositely poled regions, and on the boundary; d) vertical and lateral ND-PFM signals obtained by applying the virtual lock-in procedure to the HSDC data. Points of interest 2,4 correspond to those in Figure 1

Figure 2 shows a direct comparison of vertical and lateral c-PFM and ND-PFM signals, obtained from the same location on the calibration sample (see Supporting Information Figure S1). Note that in all cases, we display in-phase signals following a quadrature signal minimization procedure as described by Jungk et al. ${ }^{28}$. Topography of the sample could be obtained directly from the feedback loop of the AFM in both methods (points $1 \& 3$ in Figure 1, see Supporting Information Figure S1). In c-PFM, a lock-in amplifier produced the PFM signals (point 2) shown in Figure 2a. Cross-sectional line scans were taken to examine the results quantitatively for different values of $V_{\text {ac. }}$. In ND-PFM, plotting the simultaneous lock-in amplifier signal does not result in meaningful results (see Supporting Information Figure S3 and discussion), as we are specifically interested in the data embedded in the parts of the mechanical indentation cycle where the tip and sample were in contact, marked red on the high-speed blue deflection curve (Fig. 2b). Each raw force curve, corresponding to a point on the scan, provided a single value for the in-phase and quadrature (or amplitude and phase) values of the signal components at $f_{\mathrm{PFM}}$, thus enabling the construction of PFM cross-sectional line scans (Figure 2d), analogous to the c-PFM cross-sectional line scans in Figure 2c. We display the data in arbitrary units to distinguish the virtual lock-in output from the microscope hardware output; in both cases a calibration procedure is required to turn the units into meaningful deflection (pico-metre) values. It can be seen that the ND-PFM measurement yielded almost identical piezo-response information as c-PFM for our standard PPLN calibration sample. The vertical PFM signal showed the distinctive signals obtained from the two oppositely poled domains of PPLN, while the lateral signal exhibited a peak at the domain wall, arising from the opposite vertical deformation of the domains when the tip was at this location 
(Figure 2 inset). We note that the lateral signal seems less sharp compared to the c-PFM, this is probably a result of the shorter period analysed per data point, compared to the averaging time of the c-PFM lock-in amplifier. In both cases the response is linear with $V_{\mathrm{ac}}$, as expected. Overall, this demonstrates the viability and reliability of ND-PFM as a quantitative tool for the spatially resolved mapping of piezoelectricity. When comparing this result to the previously reported IC-PFM method in ambient environment (Fig. 3d-f in Ref [26]), the differences are striking, indicating that ND-PFM truly allows selective probing of the piezoelectric properties of the sample.

It should be noted that in our current implementation of ND-PFM, the piezo-response data was generated and presented in the form of line-scans rather than a 2D image. There is no fundamental restriction in the ND-PFM to achieving 2D images, and only software limitations forced us to extract and analyse the deflection data in this manner. In recent years there have been various "big data" extraction and analysis demonstrations in AFM systems, to construct 2D images of advanced data channels ${ }^{29-31}$, which could be applied to ND-PFM. Another option is to synchronize the lock-in amplifier operation with the tip-sample contact events, thus obtaining data only when it is physically valid.

\section{ND-PFM of piezoelectric polymeric and ceramic nanowires}

Next, we turn to the application of ND-PFM to samples where c-PFM imaging was not applicable. In order to demonstrate the range of piezoelectric materials that can be mapped using NDPFM, we selected both piezoelectric polymer and well as ceramic materials. First, we show measurements performed on poly-L-lactic acid (PLLA) nanowires (NWs) grown by template-wetting, and second, measurements obtained from ceramic barium zirconate titanate-barium calcium titanate (BZT-BCT) NW network. In order to increase the accuracy of the quantitative analysis, each measured material was imaged using Kelvin-probe force microscopy (KPFM), and the resultant surface potential difference was applied as bias during ND-PFM operation. This procedure was reported to significantly reduce electrostatic contributions from PFM measurements ${ }^{32}$. Furthermore, in that report, it was found that PPLN exhibits a high surface potential. We have found that is the case in our system as well, and due to inherent DC biasing limitations in our AFM system $( \pm 10 \mathrm{~V})$, we were unable to apply appropriate DC biasing during PFM of PPLN. Therefore, we have turned to using a $100 \mathrm{~nm}$ thick poly (vinylidene fluoride-trifluoroethylene) (PVDF-TrFE) film for calibration, where a conservative $d_{33}$ coefficient of $10 \mathrm{pm} \mathrm{V}^{-15}$ was used (see Supporting Information Figure S4 and related discussion).

PLLA is a piezoelectric bio-polymer ${ }^{33}$, and as such, PLLA NWs are an example of a class of materials widely investigated for flexible and bio-compatible sensors and generators ${ }^{34,35}$. PLLA exhibits shear piezoelectricity, therefore lateral PFM signals are of interest ${ }^{36}$. In this respect, a limitation of AFM operation is that lateral deflection signals are measured in the direction perpendicular to the long-axis of the cantilever, whereas lateral deflections parallel to the cantilever may couple to the vertical PFM 
signal due to cantilever buckling mode ${ }^{19}$. A thorough PFM investigation therefore usually involves a $90^{\circ}$ rotation of the sample to characterize the two perpendicular in-plane components. In our experiments, it was found that the only stable configuration, allowing c-PFM measurements of randomly dispersed PLLA NWs without their movement during scanning, was when the NW and cantilever are misaligned by $\sim 45^{\circ}$ (see Supporting Information and Ref. ${ }^{24}$ for details), thus preventing in-depth understanding of the piezoelectric domain configurations. Therefore, a successful operation of ND-PFM in this case bears immediate benefits by allowing measurement of the NWs in the parallel and the perpendicular orientations. In addition, we observed that no NWs were dislodged or damaged in ND-PFM, whereas this was not strictly the case for c-PFM.

Figure 3 shows the vertical and lateral PFM (in-phase) signals obtained from PLLA NWs lying parallel (left-hand side insets) and transverse (right-hand side insets) to the cantilever, thus enabling the measurement of lateral PFM signals in two different orientations. Scans were obtained along the NW (Supporting Information Figure S2), and across the NW (Figure 3) for both orientations, requiring rotation of the scanning direction by about $270^{\circ}$ (an operation physically different than rotation of the sample). In order to reduce electrostatic contributions to the PFM signal, prior to conducting ND-PFM measurements, Kelvin probe force-microscopy (KPFM) measurements were carried out, and the measured contact potential $(-100 \mathrm{mV})$ was applied during ND-PFM, following the procedure suggested by Kim et $a l . .^{32}$ (see Supporting Information). Figures 3a,b show the vertical PFM signals obtained from either configuration, while scanning the cantilever across the NW (the NW topography is shown for convenience). For the most part, both responses were found to be similar, with a flat signal from the substrate (which can be related to background noise), and a negative response from the NW, also mainly flat. The dips at the right hand side of the graph result from insufficient tip-NW contact at that NW edges, and were not taken into account. Figures $3 \mathrm{c}, \mathrm{d}$ show the lateral PFM signals obtained on the same scans via the second high-speed channel. Most noticeably, the signals of each orientation were distinct, whereby in the NW/cantilever transverse orientation, the signal from the two sides of the NW changed sign, compared to the parallel configuration where the signal was mostly flat. Based on the calibration procedure described in Supporting Information (Figure S4), small effective lateral piezoelectric coefficients were evaluated $\left(d_{\mathrm{eff}} \sim 0.02 \mathrm{pm} \mathrm{V}^{-1}\right)$. The small coefficient could result from the indirect relationship between the induced piezoelectric shear strain and the measured lateral point displacement in NWs (compared to out-of-plane $d_{33}$-type deformations), and the molecular orientation of the crystalline domains in the NW. This result sheds light on the piezoelectric behaviour and structure of the PLLA NW, adding information to our previous study limited to $45^{\circ}$ scan direction ${ }^{24}$. 

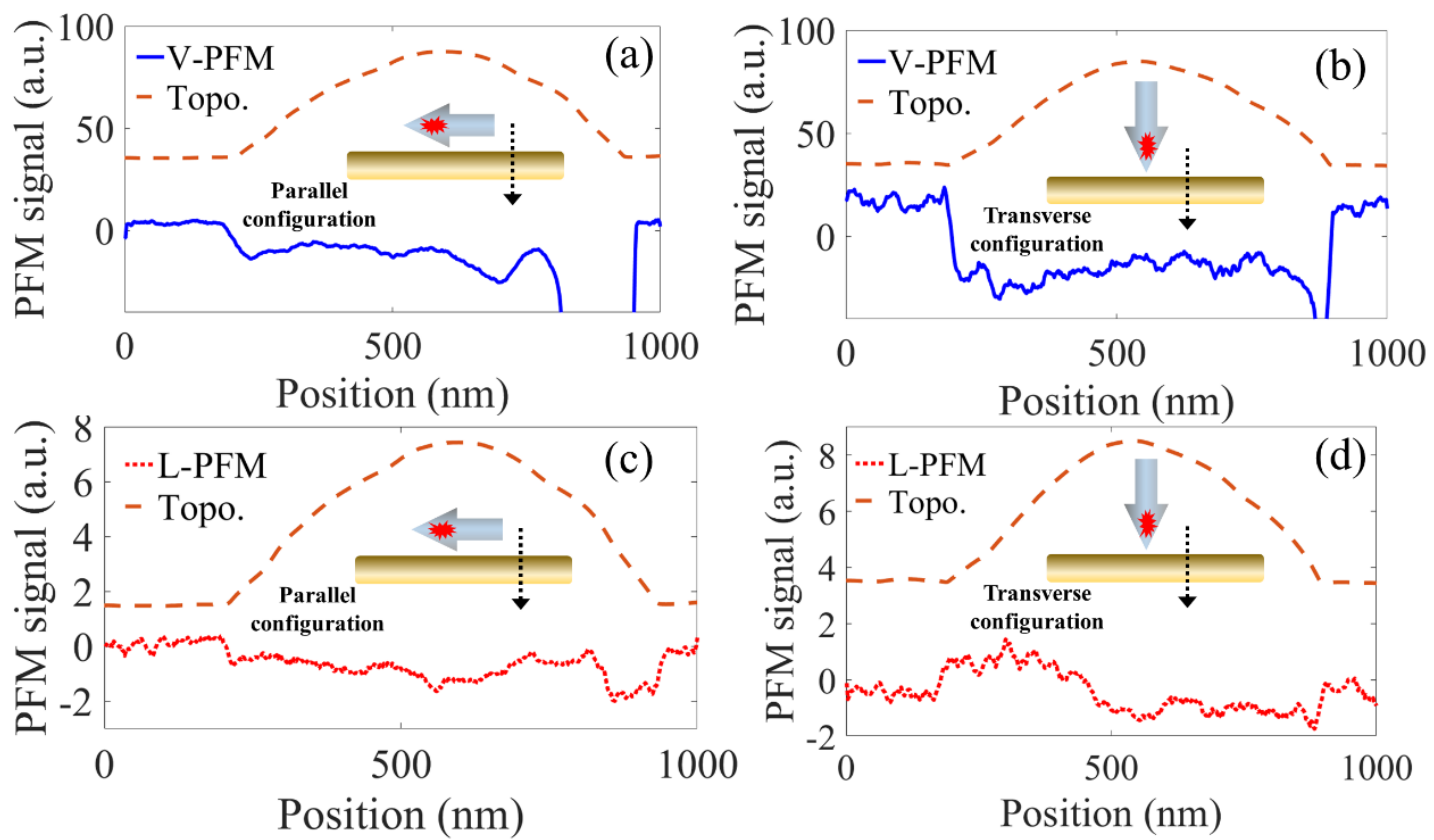

Figure 3. ND-PFM of PLLA NWs in different orientations: a,b) vertical PFM signals obtained from scanning the tip across the NW, with tip/cantilever [a] parallel, [b] transversely oriented; c,d) lateral PFM signals obtained on the same scans with [c] parallel, [d] transverse orentation. Schematic insets show the orientation of the NW relative to that of the cantilever (wide arrow with red laser spot), and the scan directions (dashed arrow).

In a recent work from our group, we studied the ferroelectric and dielectric properties of lead-free polycrystalline BCT-BZT NW network which was interestingly found to have an enhanced Curie temperature ${ }^{25}$. However, we were unable to image this NW network using c-PFM, which is an otherwise routine characterization technique for ferroelectric thin films. We found that the NW network could not sustain the pressure induced by contact-mode AFM operation (see Supporting Information Figure S5a), and we only managed to perform and report single-point spectroscopy measurements, without correlation to topography ${ }^{25}$. Several other reports also present topography images accompanied by single point spectroscopy data when studying non-planar ferroelectric materials ${ }^{37,38}$, highlighting a key deficiency in the applicability of c-PFM to nanoscale objects in particular.

Figure 4 shows the results obtained when performing ND-PFM on the BCT-BZT NW network. Figures $4 \mathrm{a}$,b show the tapping and peak-force mode topography images of the NWs, with the area where the high-speed data capture procedure was performed designated by an arrow. These NWs appeared slightly tilted relative to the substrate, and we assume the tip is scanning across their radial periphery. Figures $4 \mathrm{c}, \mathrm{d}$ show a schematic of the scan across the NWs, as well as a TEM image showing the polycrystalline nature of the NWs. Figures 4e,f show the results obtained from the ND-PFM scan. Clearly, the different NWs represented in this topography by bumps in the scan line yield different PFM responses both in the vertical and lateral signal. Considering the polycrystalline nature of these NWs, this result is to be expected, as there is no common crystalline orientation. Furthermore, it can be seen that the trends in the vertical and lateral images were decoupled, i.e. the two local peaks in the lateral 
signal corresponded to a peak and a trough in the vertical signals (marked by arrows). The PVDF-TrFE based calibration (see supporting information Figure S4) yielded a sensitivity value of $\sim 0.33$ pm/a.u. for the vertical response. Therefore, at $V_{\text {ac }}=6 \mathrm{~V}$, the $\sim 160$ a.u. difference in the vertical PFM signal, peak-to-trough, yielded an overall difference in the effective piezoelectric coefficient of about $10 \mathrm{pm} / \mathrm{V}$ obtained along the NW network. Since this measurement is comparative by nature (measuring NW to NW differences), and considering there might be a (voltage dependent) background noise present in the measurement, it is difficult to establish absolute values for the coefficient in this case. This is a general consequence of PFM operation on this sample, and not unique to the use of our method. Nonetheless, the ND-PFM provides valuable information about the piezoelectric properties of the NW network, which can be correlated to topography and crystal structure, in a more comprehensive study.

In order to further validate the measurements, the same scan was subsequently repeated and analysed, for both the vertical and lateral signals, where a high degree of reproducibility was demonstrated (Supporting Information Figure S5b). In addition, a different region of the sample, several microns away, was also scanned and resulted in similar characteristics (Supporting Information Figure $\mathrm{S} 5 \mathrm{c})$.

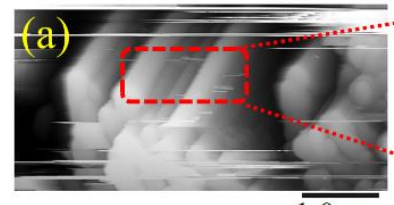

$\overline{1.0 \mu \mathrm{m}}$

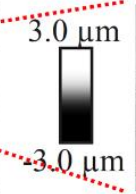

.3. $0 . \mu \mathrm{m}$

200

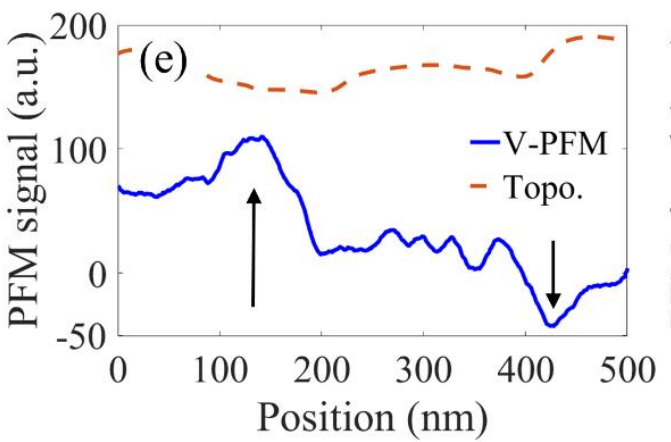

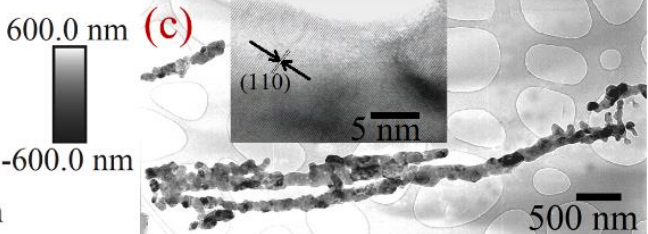

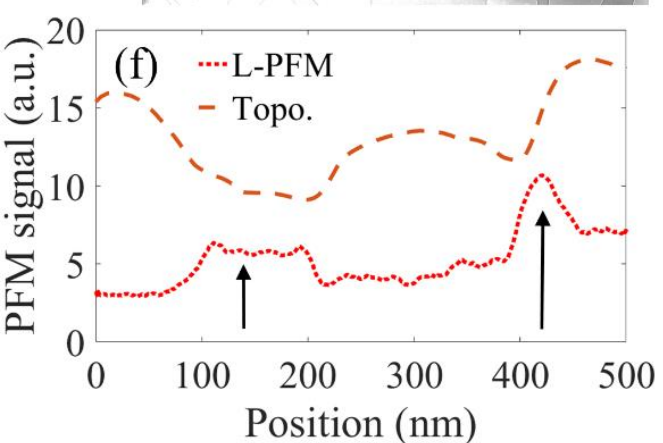

Figure 4. ND-PFM of BCT-BZT NW network: a) tapping mode topography, with the area selected for PFM marked by dashed rectangle; b) peak-force mode topography of the selected area, with the area where the HSDC procedure was performed; c) TEM image of a NW showing its polycrystalline nature, the inset shows a close up to a single grain; d) schematic of the scan over the polycrystalline NWs; e) topography and vertical PFM signal obtained from the different NWs; f) topography and lateral PFM signal obtained from the NWs. 


\section{Conclusions}

In summary, we have developed a non-destructive PFM method based on discontinuous tip-sample contact that allows for direct measurements of spatially resolved piezoelectric properties of nanomaterials. Measurements on relatively flat samples such as PPLN and PVDF-TrFE thin films have validated the method, with results comparable to conventional c-PFM, for example when resolving the lateral PFM signal peak in domain boundaries of PPLN. The ND-PFM was then applied to sensitive piezoelectric NW samples, both polymeric and ceramic in nature, enabling the extraction of data unavailable through c-PFM. In the case of PLLA NWs, scanning orientations which resulted in movement of the NW were proven possible, resulting in distinct lateral signals when the tip is parallel/transverse to the NW. This work is particularly significant given the recent interest in soft piezoelectric biological or bio-compatible materials, motivated by medical applications ${ }^{24,39}$. In the case of a BCT-BZT NW network, ND-PFM allowed acquiring PFM data related to the sample topography, whereas scanning in contact-mode was found to be destructive. Such measurements provide new insight into how microstructural features affect piezoelectricity at the nanoscale, enabling finer control over the tuning of these properties. ND-PFM can therefore be used to map piezoelectricity in nanoscale systems that were previously inaccessible through c-PFM but that are technologically important, for example, in our work on piezoelectricity in semiconducting III-V NWs ${ }^{40}$ where single-NW PFM measurements have proven to be otherwise elusive. We believe that the ND-PFM technique will open up new possibilities related to piezoelectricity at the nanoscale, and will quite literally add another crucial dimension to the ferro/piezoelectric materials research community, thus extending the application of these nanomaterials to new research areas.

\section{Methods}

PFM setup and measurements. All experiments were conducted using a Multimode VIII by Bruker with a Nanoscope $V$ controller. The tips used were MESP-RC-V2 also by Bruker. The AC frequency used as PFM signal was $125 \mathrm{kHz}$ with voltages ranging from 2-8 V. The built in High Speed Data Capture (HSDC) procedure was used to extract the vertical and lateral tip deflection.

Data Analysis. Raw high speed deflection data was processed and analysed using self-written MATLAB code. Main sections of code are available (see Data Availability statement below).

PLLA NW synthesis. Full description can be found in Ref. ${ }^{24}$; briefly, the PLLA nanowires were fabricated using a variation on the template wetting technique. This involved floating a silver-capped thin nanoporous anodized aluminium oxide (AAO) membrane (or 'template', $60 \mu \mathrm{m}$ thick, $200 \mathrm{~nm}$ pore diameter) onto a heated solution of PLLA and allowing the solution to infiltrate the pores via capillary action. Subsequently, the template was removed from the droplet and any residual solvent was removed 
using a doctor blade method. (Inevitably, a thin PLLA film $(<0.5 \mu \mathrm{m})$ would remain on the infiltrated template surface. The silver capping layer prevents a PLLA film from forming on the uppermost surface. The silver layer was removed following infiltration using aqueous ferric nitrate $\left(\mathrm{Fe}\left(\mathrm{NO}_{3}\right)_{3}\right)$, $1 \mathrm{M})$, thus allowing the AAO template to be selectively etched. The fragile NW film was then floated onto Si wafer and transferred to de-ionised water for washing several times. Following thorough drying at room temperature, the nanowires were dispersed in isopropyl alcohol to be cast onto substrates for PFM measurements.

BCT-BZT NWs synthesis. Full description can be found in Ref. ${ }^{25}$; briefly: BCT-0.5BZT sol was prepared using barium acetate, calcium acetate, titanium (IV) isopropoxide and zirconyl oxychloride octahydrate in ethanol. Then, PI porous templates from ipPore (it4ip) of $20 \mu \mathrm{m}$ thickness and a $200 \mathrm{~nm}$ average pore diameter were used for infiltration of BCT-0.5BZT sol. The sol prepared above was then drop-casted onto the template and vacuum infiltrated within the pores. The sol containing the $\mathrm{Ba}, \mathrm{Ca}$, $\mathrm{Ti}$ and $\mathrm{Zr}$ precursors was allowed to infiltrate and polymerize within the pores of polyimide membrane after which they were dried and annealed at $1000{ }^{\circ} \mathrm{C}$ for 24 hours placing the polymide templates of $\mathrm{Au}$ coated silicon substrates. High temperature annealing lead to the decomposition of the polyimide membrane and a white powdery film remained on the Au coated Si substrates. A BCT-0.5BZT NW network was thus obtained, which was used for PFM analyses.

BCT-BZT NWs TEM. Microstructural studies were carried out by transmission electron microscopy (HRTEM, JEOL 4000EXII). For TEM, NWs were scrapped off from the substrates and were dispersed in ethanol just my gentle shaking in order to avoid breakage of the polycrystalline NWs. A few drops of dispersant were then placed on C coated 400 mesh TEM grids (Ted Pella Inc.) and was air dried for final observation.

Data Availability. Raw data for figures, as well as major code sections are available at the University of Cambridge open data repository, Dspace.

\section{References}

1. Gao, Y.; Wang, Z. L., Electrostatic potential in a bent piezoelectric nanowire. The fundamental theory of nanogenerator and nanopiezotronics. Nano Letters 2007, 7 (8), 2499-2505.

2. $\quad$ Minary-Jolandan, M.; Bernal, R. A.; Kujanishvili, I.; Parpoil, V.; Espinosa, H. D., Individual GaN Nanowires Exhibit Strong Piezoelectricity in 3D. Nano Letters 2012, 12 (2), 970-976.

3. $\quad$ Wu, Y. J.; Gu, Q. Z.; Ding, G. Z.; Tong, F. Q.; Hu, Z. J.; Jonas, A. M., Confinement Induced Preferential Orientation of Crystals and Enhancement of Properties in Ferroelectric Polymer Nanowires. Acs Macro Letters 2013, 2 (6), 535-538.

4. Cauda, V.; Torre, B.; Falqui, A.; Canavese, G.; Stassi, S.; Bein, T.; Pizzi, M., Confinement in Oriented Mesopores Induces Piezoelectric Behavior of Polymeric Nanowires. Chemistry of Materials 2012, 24 (21), 4215-4221. 
5. Calahorra, Y.; Whiter, R. A.; Jing, Q. S.; Narayan, V.; Kar-Narayan, S., Localized electromechanical interactions in ferroelectric $\mathrm{P}(\mathrm{VDF}-\mathrm{TrFE})$ nanowires investigated by scanning probe microscopy. APL Materials 2016, 4 (11), 116106.

6. $\quad$ Kvasov, A.; McGilly, L. J.; Wang, J.; Shi, Z. Y.; Sandu, C. S.; Sluka, T.; Tagantsev, A. K.; Setter, N., Piezoelectric enhancement under negative pressure. Nature Communications 2016, 7.

7. Wang, Z. L.; Song, J. H., Piezoelectric nanogenerators based on zinc oxide nanowire arrays. Science 2006, 312 (5771), 242-246.

8. Qi, Y.; Kim, J.; Nguyen, T. D.; Lisko, B.; Purohit, P. K.; McAlpine, M. C., Enhanced Piezoelectricity and Stretchability in Energy Harvesting Devices Fabricated from Buckled PZT Ribbons. Nano Letters 2011, 11 (3), 1331-1336.

9. Whiter, R. A.; Narayan, V.; Kar-Narayan, S., A Scalable Nanogenerator Based on Self-Poled Piezoelectric Polymer Nanowires with High Energy Conversion Efficiency. Advanced Energy Materials 2014, 4 (18), 1400519.

10. Boughey, F. L.; Davies, T.; Datta, A.; Whiter, R. A.; Sahonta, S. L.; Kar-Narayan, S., Vertically aligned zinc oxide nanowires electrodeposited within porous polycarbonate templates for vibrational energy harvesting. Nanotechnology 2016, 27 (28), 28 LT02.

11. Ou, C.; Sanchez-Jimenez, P. E.; Datta, A.; Boughey, F. L.; Whiter, R. A.; Sahonta, S. L.; KarNarayan, S., Template-Assisted Hydrothermal Growth of Aligned Zinc Oxide Nanowires for Piezoelectric Energy Harvesting Applications. ACS Applied Materials \& Interfaces 2016, 8 (22), 13678-13683.

12. Wang, X. D.; Zhou, J.; Song, J. H.; Liu, J.; Xu, N. S.; Wang, Z. L., Piezoelectric field effect transistor and nanoforce sensor based on a single $\mathrm{ZnO}$ nanowire. Nano Letters 2006, 6 (12), 2768-2772. 13. Yu, B. W.; Fu, Y. M.; Wang, P. L.; Zhao, Y. Y.; Xing, L. L.; Xue, X. Y., Enhanced piezohumidity sensing of a $\mathrm{Cd}-\mathrm{ZnO}$ nanowire nanogenerator as a self-powered/active gas sensor by coupling the piezoelectric screening effect and dopant displacement mechanism. Physical Chemistry Chemical Physics 2015, 17 (16), 10856-10860.

14. Pan, C. F.; Dong, L.; Zhu, G.; Niu, S. M.; Yu, R. M.; Yang, Q.; Liu, Y.; Wang, Z. L., Highresolution electroluminescent imaging of pressure distribution using a piezoelectric nanowire LED array. Nature Photonics 2013, 7 (9), 752-758.

15. Gruverman, A.; Kalinin, S. V., Piezoresponse force microscopy and recent advances in nanoscale studies of ferroelectrics. Journal of Materials Science 2006, 41 (1), 107-116.

16. Kolosov, O.; Gruverman, A.; Hatano, J.; Takahashi, K.; Tokumoto, H., NANOSCALE VISUALIZATION AND CONTROL OF FERROELECTRIC DOMAINS BY ATOMIC-FORCE MICROSCOPY. Physical Review Letters 1995, 74 (21), 4309-4312.

17. Rodriguez, B. J.; Gruverman, A.; Kingon, A. I.; Nemanich, R. J.; Ambacher, O., Piezoresponse force microscopy for polarity imaging of GaN. Applied Physics Letters 2002, 80 (22), 4166-4168.

18. Guthner, P.; Dransfeld, K., LOCAL POLING OF FERROELECTRIC POLYMERS BY SCANNING FORCE MICROSCOPY. Applied Physics Letters 1992, 61 (9), 1137-1139.

19. Kalinin, S. V.; Rodriguez, B. J.; Jesse, S.; Shin, J.; Baddorf, A. P.; Gupta, P.; Jain, H.; Williams, D. B.; Gruverman, A., Vector piezoresponse force microscopy. Microscopy and Microanalysis 2006, 12 (3), 206-220.

20. Jungk, T.; Hoffmann, A.; Soergel, E., Detection mechanism for ferroelectric domain boundaries with lateral force microscopy. Applied Physics Letters 2006, 89 (4), 3.

21. Rodriguez, B. J.; Gruverman, A.; Kingon, A. I.; Nemanich, R. J.; Cross, J. S., Threedimensional high-resolution reconstruction of polarization in ferroelectric capacitors by piezoresponse force microscopy. Journal of Applied Physics 2004, 95 (4), 1958-1962.

22. Jesse, S.; Baddorf, A. P.; Kalinin, S. V., Switching spectroscopy piezoresponse force microscopy of ferroelectric materials. Applied Physics Letters 2006, 88 (6).

23. Feigl, L.; Sluka, T.; McGilly, L. J.; Crassous, A.; Sandu, C. S.; Setter, N., Controlled creation and displacement of charged domain walls in ferroelectric thin films. Scientific Reports 2016, 6.

24. Smith, M.; Calahorra, Y.; Jing, Q.; Kar-Narayan, S., Direct observation of shear piezoelectricity in poly-l-lactic acid nanowires. APL Materials, 2017; Vol. 5, p 074105.

25. Datta, A.; Sanchez-Jimenez, P. E.; Al Rahal Al Orabi, R.; Calahorra, Y.; Ou, C.; Sahonta, S.L.; Fornari, M.; Kar-Narayan, S., Lead-Free Polycrystalline Ferroelectric Nanowires with Enhanced Curie Temperature. Advanced Functional Materials 2017, 1701169. 
26. Rodriguez, B. J.; Jesse, S.; Habelitz, S.; Proksch, R.; Kalinin, S. V., Intermittent contact mode piezoresponse force microscopy in a liquid environment. Nanotechnology 2009, 20 (19).

27. Pittenger, B.; Erina, N.; Su, C., Quantitative Mechanical Property Mapping at the Nanoscale with PeakForce QNM. In Application Note AN128, Bruker: 2010.

28. Jungk, T.; Hoffmann, A.; Soergel, E., Consequences of the background in piezoresponse force microscopy on the imaging of ferroelectric domain structures. Journal of Microscopy-Oxford 2007, 227 (1), 72-78.

29. Belianinov, A.; Kalinin, S. V.; Jesse, S., Complete information acquisition in dynamic force microscopy. Nature Communications 2015, 6.

30. Collins, L.; Belianinov, A.; Somnath, S.; Rodriguez, B. J.; Balke, N.; Kalinin, S. V.; Jesse, S., Multifrequency spectrum analysis using fully digital $G$ Mode-Kelvin probe force microscopy. Nanotechnology 2016, 27 (10).

31. Collins, L.; Ahmadi, M.; Wu, T.; Hu, B.; Kalinin, S. V.; Jesse, S., Breaking the Time Barrier in Kelvin Probe Force Microscopy: Fast Free Force Reconstruction Using the G-Mode Platform. ACS Nano 2017.

32. Kim, S.; Seol, D.; Lu, X. L.; Alexe, M.; Kim, Y., Electrostatic-free piezoresponse force microscopy. Scientific Reports 2017, 7.

33. Fukada, E., BIOELECTRETS AND BIOPIEZOELECTRICITY. Ieee Transactions on Electrical Insulation 1992, 27 (4), 813-819.

34. Cauda, V.; Canavese, G.; Stassi, S., Nanostructured piezoelectric polymers. Journal of Applied Polymer Science 2015, 132 (13), 14.

35. Ribeiro, C.; Sencadas, V.; Correia, D. M.; Lanceros-Mendez, S., Piezoelectric polymers as biomaterials for tissue engineering applications. Colloids and Surfaces B-Biointerfaces 2015, 136, 4655 .

36. Ochiai, T.; Fukada, E., Electromechanical properties of poly-L-lactic acid. Japanese Journal of Applied Physics Part 1-Regular Papers Short Notes \& Review Papers 1998, 37 (6A), 3374-3376.

37. $\quad$ Chen, Z.; Huang, J. Y.; Yang, Y. F.; Wang, Y.; Wu, Y. J.; He, H. P.; Wei, X. Y.; Ye, Z. Z.; Zeng, H. R.; Cong, H. L.; Jiang, Z. Y., Piezoelectric properties of rhombic LiNbO3 nanowires. Rsc Advances 2012, 2 (19), 7380-7383.

38. Zhou, Z.; Bowland, C. C.; Malakooti, M. H.; Tang, H. X.; Sodano, H. A., Lead-free $0.5 \mathrm{Ba}(\mathrm{Zr} 0.2 \mathrm{Ti0} .8) \mathrm{O}-3-0.5(\mathrm{Ba} 0.7 \mathrm{Ca} 0.3) \mathrm{TiO} 3$ nanowires for energy harvesting. Nanoscale 2016, $8(9)$, 5098-5105.

39. Torculas, M.; Medina, J.; Xue, W.; Hu, X., Protein-Based Bioelectronics. Acs Biomaterials Science \& Engineering 2016, 2 (8), 1211-1223.

40. Calahorra, Y.; Guan, X.; Halder, N. N.; Smith, M.; Cohen, S.; Ritter, D.; Penuelas, J.; KarNarayan, S., Exploring piezoelectric properties of III-V nanowires using piezo-response force microscopy. Semiconductor Science and Technology 2017, 32 (7), 074006.

\section{Acknowledgements}

This work was financially supported by a grant from the European Research Council through an ERC Starting Grant (Grant no. ERC-2014-STG-639526, NANOGEN). S.K-N., Y.C and A.D. are grateful for financial support from this same grant. M.S is grateful for studentship funding through the Cambridge Commonwealth, European \& International Trust.

\section{Author Contributions}

Y.C. and S.K-N. conceived the idea. Y.C. performed all AFM based measurements and data analysis. M.S. synthesized and fabricated PLLA NW samples. A.D. synthesized and fabricated BCT-BZT NW 
samples, and performed TEM measurements. Y.C. and H.B. designed and implemented the virtual lock-in code. Y.C. and S.K-N wrote the paper; all authors commented and edited the paper.

\section{Additional information}

Supplementary Information accompanies this paper

\section{Competing financial interests}

The authors declare no competing financial interests. 